\title{
The Importance of Concurrent Chemotherapy for T1 Esophageal Cancer: Role of FDG-PET/CT for Local Control
}

\author{
ICHIRO OGINO ${ }^{1}$, SHIGENOBU WATANABE ${ }^{1}$, KINGO HIRASAWA ${ }^{2}$, \\ TOSHIHORO MISUMI ${ }^{3}$, MASAHARU HATA ${ }^{4}$ and CHIKARA KUNISAKI ${ }^{5}$ \\ ${ }^{1}$ Department of Radiation Oncology, ${ }^{2}$ Division of Endoscopy, and ${ }^{5}$ Department of Surgery, \\ Gastroenterological Center, Yokohama City University Medical Center, Yokohama, Japan; \\ ${ }^{3}$ Department of Biostatistics, Yokohama City University, Yokohama, Japan; \\ ${ }^{4}$ Division of Radiation Oncology, Department of Oncology, \\ Yokohama City University Graduate School of Medicine, Yokohama, Japan
}

\begin{abstract}
Aim: To evaluate whether patients with $T 1$ esophageal squamous cell carcinoma receiving definitive radiotherapy can be managed without concurrent chemotherapy, and the role of ${ }^{18}$ F-fluorodeoxyglucose positron-emission tomography with computed tomography (FDG-PET/CT) in demonstrating local control (LC). Patients and Methods: Twenty-four out of 37 patients with newlydiagnosed $T 1$ EC treated with definitive radiotherapy between July 2009 and July 2016 were retrospectively analyzed. FDGPET/CT was performed before treatment. Eleven patients were assigned to a concurrent chemoradiotherapy (CRT) group. Thirteen were placed in a no-CRT group. The two groups were compared and univariate analysis of clinical factors influencing the prognosis in each group was conducted. Results: Mean radiotherapy doses were $59.2 \mathrm{~Gy}$ in the noCRT group and $55.5 \mathrm{~Gy}$ in the CRT group $(p=0.025)$. Overall survival, disease-free survival, and $L C$ rates at 2 years were lower in the no-CRT group compared to the CRT group. Disease-free survival and $L C$ rates at 2 years were significantly lower in the patients with FDG-avid primary tumor in the no-CRT group ( $p=0.002$ and $p=0.002$, respectively). All patients with FDG-avid primary tumors in the no-CRT group developed local recurrence. Conclusion: It is important to note that all patients with FDG-avid primary tumor in the no-CRT group developed local recurrence. This
\end{abstract}

This article is freely accessible online.

Correspondence to: Ichiro Ogino, MD, Department of Radiation Oncology, Yokohama City University Medical Center, 4-57 Urahune-cho, Minami-ku, Yokohama, Kanagawa Prefecture, Japan. Tel: +81 452615656, e-mail: ogino1ro@urahp.yokohama-cu.ac.jp

Key Words: Local control, T1 esophageal cancer, concurrent chemoradiotherapy, FDG-PET/CT. would suggest that concurrent chemotherapy is an integral part of disease management in patients with T1 esophageal squamous cell carcinoma.

Although the majority of patients with esophageal cancer (EC) presented with locally advanced disease in historical series, more recent series show that with increased surveillance endoscopy, many patients (18-33\%) are being diagnosed with early-stage lesions $(1,2)$. T1 EC is defined as a malignant tumor that is limited to the lamina propria mucosa or muscularis mucosa (stage T1a), or submucosa (stage T1b) in the eighth edition of the American Joint Committee on Cancer/Union for International Cancer Control TNM staging system (3).

${ }^{18}$ F-FIuorodeoxyglucose positron-emission tomography with computed tomography (FDG-PET/CT) is important for staging EC and selecting patients for surgery. FDG-PET/CT is also helpful for evaluating the treatment response and detecting recurrent disease throughout the entire body during follow-up (4). Although FDG-PET/CT plays a crucial role, few studies have evaluated its clinical significance for patients with T1 EC treated with radiotherapy.

There was a significant reduction in local recurrence in patients treated with concurrent chemoradiotherapy (CRT) compared with those treated with radiotherapy alone $(5,6)$. However, higher rates of CRT discontinuation and toxicity in elderly patients were observed (7). We managed elderly patients aged $\geq 75$ years with sequential radiotherapy and chemotherapy; for patients unable to tolerate chemotherapy, we apply radiotherapy alone and we administer CRT to nonelderly patients with T1 EC.

The aims of this retrospective study were to investigate whether patients with $\mathrm{T} 1 \mathrm{EC}$ receiving definitive radiotherapy can be managed without concurrent chemotherapy and to assess the role of FDG-PET/CT for these patients in demonstrating local control (LC). 


\section{Patients and Methods}

Patients. Thirty-seven patients with newly diagnosed T1 EC were treated with definitive radiotherapy between July 2009 and July 2016. Patients were referred for definitive radiotherapy either due to their preference, or because it was considered that they were medically unfit for surgery. Patients who had definitive CRT for synchronous double cancer of the esophagus and head and neck carcinomas, or endoscopic submucosal dissection before radiotherapy were excluded from the study. Twenty-four patients therefore underwent the retrospective analysis.

Pretreatment evaluation. The pretreatment evaluation consisted of a complete history and physical examination, blood counts, chemistry profile, esophagography, esophagoscopy and contrastenhanced thoracoabdominal CT.

Disease in all patients was staged with FDG-PET/CT before radiation planning. Most images were obtained at the Universityaffiliated PET Institute using a Biograph $16^{\circledR}$ TruePoint (Siemens Medical Solutions USA Inc., Malvern, PA, USA). After fasting for $6 \mathrm{~h}$, FDG was intravenously injected based on the patient's weight $(150-300 \mathrm{MBq})$ when the blood glucose level was $<200 \mathrm{mg} / \mathrm{dI}$. Sixty minutes after the FDG injection, non-contrast CT with $110 \mathrm{mAs}, 130 \mathrm{kV}$, and a $5-\mathrm{mm}$ slice thickness was conducted, followed by a PET emission scan. PET images were reconstructed iteratively (OS-EM algorithm; 2 iterations, 14 subsets) with a matrix size of $168 \times 168$.

Magnifying endoscopy with narrow-band imaging (ME-NBI) and Lugol chromoendoscopy was performed after conventional endoscopy. Lugol-unstained regions of $\geq 5 \mathrm{~mm}$ were subjected to biopsies, and unstained regions were marked cranially and caudally with metallic endoclips. All patients had histologically confirmed squamous cell carcinoma (SCC) of the esophagus.

Treatment groups. Eleven patients were assigned to a concurrent chemoradiotherapy (CRT) group. Thirteen were placed in a no-CRT group. Sequential chemotherapy (SCT) was scheduled for the noCRT group unless patients were medically unfit for chemotherapy. For the CRT group, SCT was added when the response of the primary tumor was not complete.

Three-dimensional conformal radiotherapy involved 15-MV photons administered in $5 \times 1.8$-to 2.0 -Gy fractions per week. The primary gross tumor volume (GTV) was set as the esophagus between the two marked endoclips. All the lesions detected by FDG-PET/CT were in the GTV. The clinical target volume (CTV) encompassed the GTV with 4-cm craniocaudal margins and regional lymph nodes (LN). Elective supraclavicular LN regions were included when the proximal tumor was above the carina level. Elective abdominal LN regions were included when the distal tumor was near the gastroesophageal junction. The planning tumor volume (PTV) was derived from the CTV plus $1 \mathrm{~cm}$. The primary dose was delivered at 40-45 Gy to the PTV. The boost PTV was derived from the boost CTV (GTV with $2-\mathrm{cm}$ craniocaudal and $0.5-\mathrm{cm}$ radial margins) plus $0-1 \mathrm{~cm}$. Involved nodes with $0.5-\mathrm{cm}$ margins were also contained in the boost CTV (8).

Two cycles of combined 5-fluorouracil and cisplatin were used for patients treated with CRT $\left(700 \mathrm{mg} / \mathrm{m}^{2} 5\right.$-fluorouracil and $70 \mathrm{mg} / \mathrm{m}^{2}$ cisplatin administered on days $1-4$, and day 1 , respectively). For SCT, two cycles of combined 5-fluorouracil and cisplatin comprised the regimen for the no-CRT group $\left(800 \mathrm{mg} / \mathrm{m}^{2} 5\right.$-fluorouracil and
$80 \mathrm{mg} / \mathrm{m}^{2}$ cisplatin on days $1-5$, and day 1 , respectively) and two cycles of combined docetaxel and cisplatin comprised the regimen for the CRT group $\left(60 \mathrm{mg} / \mathrm{m}^{2}\right.$ docetaxel on days 1 and 21 , and $60 \mathrm{mg} / \mathrm{m}^{2}$ cisplatin on days 1 and 21).

Follow-up and statistical analysis. The endoscopic response was assessed at 1 month after radiotherapy. Follow-up examinations including endoscopy and enhanced CT were conducted at 3-to 6month intervals for 2 years, and were scheduled according to individual clinical findings after the completion of radiotherapy. Local recurrence was determined based on pathological signs of vital tumor tissue.

Groups were compared using the chi-squared (Fisher's exact) test for frequencies and $t$-test for continuous variables. Overall survival (OS), disease-free survival (DFS), and LC rates were calculated from the date of starting radiotherapy. Kaplan-Meier curves were used for univariate analyses to assess the variables as risk predictors and compared using the log-rank test. Factors with a $p$-value of <less than 0.05 were considered significant. Statistical analysis was performed with SPSS version 25.0 (IBM Corp., Armonk, NY, USA).

\section{Results}

Esophageal tumors were detected by endoscopic screening in 17 asymptomatic patients (Table I). Six patients were without any evidence of other disease, and in 11 cases EC was detected during follow-up for another disease. Seven other patients presented with mild symptoms including dysphagia and odynophagia. All patients had albumin $>3.6 \mathrm{mg} / \mathrm{dl}$, and C-reactive protein $<0.7 \mathrm{mg} / \mathrm{dl}$, and no weight loss. Primary tumor was not detected by esophagography or contrastenhanced CT in all patients.

There were no severe complications from therapy. No patient had a delay in radiotherapy exceeding 5 days. The OS, DFS, and LC rates of the 24 patients at 2 years by Kaplan-Meier product-limited methods were 82.1, 62.5, and $75 \%$, respectively. Of 10 recurrences, three patients had LN recurrence without primary recurrence and seven had recurrence only at the primary site within radiation fields. All LN metastases were outside radiotherapy fields. Local recurrence developed 2-41 months (median=8 months) after radiotherapy.

Comparison of the no-CRT and CRT groups. The distribution of clinical factors and comparison of the two groups are shown in Table I. Significant differences between the noCRT group and CRT group included the mean age ( 74 years vs. 64.5 years, respectively, $p=0.016$ ) and mean applied cumulative radiotherapy dose to the primary tumor $(59.2 \mathrm{vs}$. $55.5 \mathrm{~Gy}, p=0.025$, respectively). Out of eight deaths, five were due to EC. One patient in the no-CRT group and two in the CRT group died of other diseases without any recurrence. The 2-year OS, DFS and LC rates tended to be higher (non-significantly so) in the CRT group than the noCRT group (Figure 1). The maximum standardized uptake value (SUVmax) of FDG-avid primary tumors ranged from 
Table I. Baseline clinical characteristics of all patients with esophageal cancer, treated with and without concurrent chemotherapy (CRT).

\begin{tabular}{|c|c|c|c|c|c|}
\hline Characteristic & & All $(n=24)$ & No-CRT (n=13) & CRT $(\mathrm{n}=11)$ & $p$-Value \\
\hline Age, years & Median (range) & $73(43-81)$ & $77(43-81)$ & $65(53-74)$ & 0.016 \\
\hline \multirow[t]{2}{*}{ Gender, n (\%) } & Female & $2(8.3)$ & $1(7.7)$ & $1(9.1)$ & $>0.99$ \\
\hline & Male & $22(91.7)$ & $12(92.3)$ & $10(90.9)$ & \\
\hline \multirow[t]{2}{*}{ Lymph node involvement, n (\%) } & No & $21(87.5)$ & $11(84.6)$ & $10(90.9)$ & $>0.99$ \\
\hline & Yes & $3(12.5)$ & $2(15.4)$ & $1(9.1)$ & \\
\hline \multirow[t]{4}{*}{ Tumor site, $\mathrm{n}(\%)$} & Cervical & $1(0)$ & $1(4.2)$ & $0(7.7)$ & $0.158^{*}$ \\
\hline & Upper thoracic & $2(18.2)$ & $0(8.3)$ & $2(0)$ & \\
\hline & Mid-thoracic & $15(45.5)$ & $10(62.5)$ & $5(76.9)$ & \\
\hline & Lower thoracic & $6(36.4)$ & $2(25)$ & $4(15.4)$ & \\
\hline Tumor length, $\mathrm{cm}$ & Median (range) & $6.1(2-16.5)$ & $5.5(2-12.8)$ & $10(2-16.5)$ & 0.053 \\
\hline \multirow[t]{2}{*}{ FDG-avid primary tumor, n (\%) } & No & $14(58.3)$ & $9(69.2)$ & $5(45.5)$ & 0.408 \\
\hline & Yes & $10(41.7)$ & $4(30.8)$ & $6(54.5)$ & \\
\hline \multirow[t]{2}{*}{ Symptomatic, n (\%) } & No & $17(70.8)$ & $10(76.9)$ & $7(63.6)$ & 0.659 \\
\hline & Yes & $7(29.2)$ & $3(23.1)$ & $4(36.4)$ & \\
\hline Applied radiation dose (Gy) & Median (range) & $59.4(50.4-64)$ & $59.4(50.4-64)$ & $54(50.4-59.4)$ & 0.025 \\
\hline Follow-up period, months & Median (range) & $26.5(14-64)$ & $26(18-40)$ & $38(14-64)$ & 0.058 \\
\hline
\end{tabular}

FDG: ${ }^{18}$ F-Fluorodeoxyglucose. *Pearson's Chi-squared test.

2.8 to 5.1 , with a mean of 3.7 and median of 3.6. The mean SUVmax was 3.5 (range=3.2-3.7) for the no-CRT group and 3.8 (range $=2.8-5.1)$ for the CRT group $(p=0.491)$.

Univariate analysis of each group. Univariate analysis of clinical factors potentially influencing the OS, DFS, and LC was conducted for the no-CRT group (Table II). DFS and $\mathrm{LC}$ at 2 years were significantly lower in patients with FDG-avid primary tumor in the no-CRT group, also presented in Figure 2 ( $p=0.002$ and $p=0.002$, respectively). All four patients with FDG-avid primary tumors in the noCRT group developed local recurrence. Rates of OS at 2 years were significantly higher in patients with SCT in the no-CRT group $(p=0.026)$.

Univariate analysis of the same factors influencing the OS, DFS, and LC was also performed for the CRT group. No factor selected from Table II affected OS, DFS, or LC in the CRT group. The 2-year OS, DFS, and LC rates in the CRT group were 100,80 and $80 \%$, respectively, for patients without FDG-avid primary tumor and 80, 83.3 and $100 \%$, respectively, for those with FDG-avid primary tumor $(p=0.746, p=0.599$ and $p=0.949)$.

\section{Discussion}

It is difficult to report a large number of patients treated with definitive radiotherapy or CRT for T1N0 esophageal SCC because the National Comprehensive Cancer Network (NCCN) guidelines (version 3) recommend esophagectomy for those medically fit for surgery and endoscopic resection for patients with intramucosal carcinoma $\leq 2 \mathrm{~cm}$.
For patients with T1 EC, definitive radiotherapy and CRT have been reported as therapeutic alternatives (9-13). Sample sizes of single institutes were generally small and required multicenter or long-term sample collection. Those studies were non-comparative case series, with varying treatment protocols. The treatment field and dose of external radiotherapy show variation (13). Additionally, administrations of chemotherapy were different and some studies included data on patients treated with endoluminal brachytherapy or endoscopic submucosal dissection (10-13). Most of these series involved patients primarily with SCC. Nemoto et al. reported 78 patients with T1 EC who received external radiotherapy without endoluminal brachytherapy at nine radiotherapy institutions. All patients had SCC and were treated with or without chemotherapy. Their OS and LC rates at 2 years were 73 and $79 \%$, respectively (13). This is compatible with the present results, in which the rates were 82.1 and $75 \%$, respectively.

A pivotal randomized controlled trial (RTOG-85-01) was performed involving 123 patients. Patients had SCC (82\%) or adenocarcinoma (18\%) of the esophagus staged T1-3 N0-1 M0. CRT consisting of cisplatin and 5-fluorouracil infusions with a radiation dose of 50 Gy was compared with radiation therapy only with $64 \mathrm{~Gy}$. The 2-year OS rate for these treated with CRT was $36 \%$ compared to $10 \%$ following radiotherapy alone. Life-threatening acute toxic effects were more marked with CRT $(10 \%)$ than radiotherapy only $(2 \%)(6)$. However, the results of that study may not correspond to T1 EC. There has been no randomized study comparing patients with T1 EC treated with CRT and radiotherapy alone as far as we are aware. In our study, OS, DFS, and LC rates at 2 years tended to be higher in the CRT group than the no-CRT group. 
A

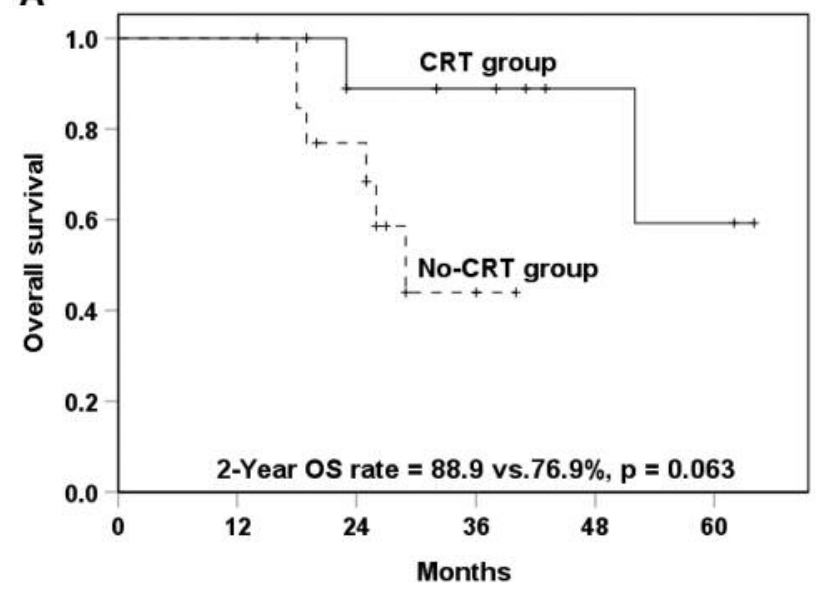

B

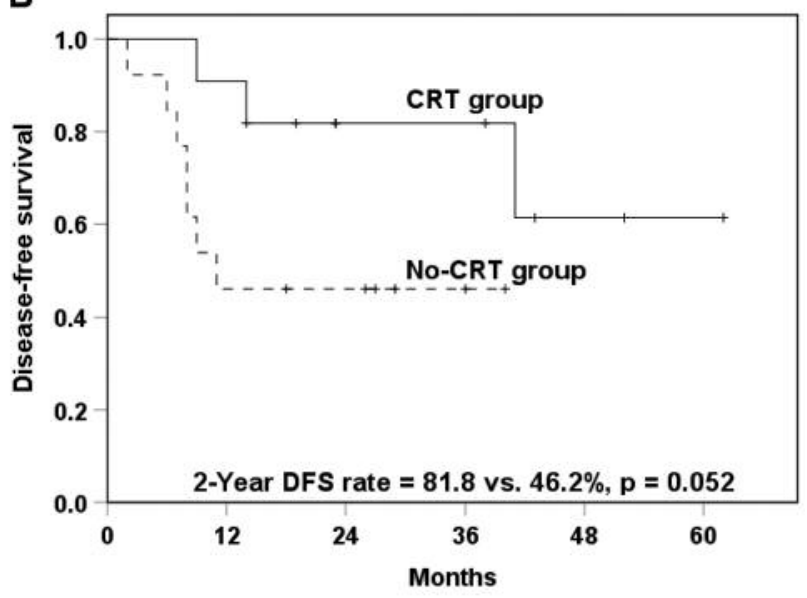

C

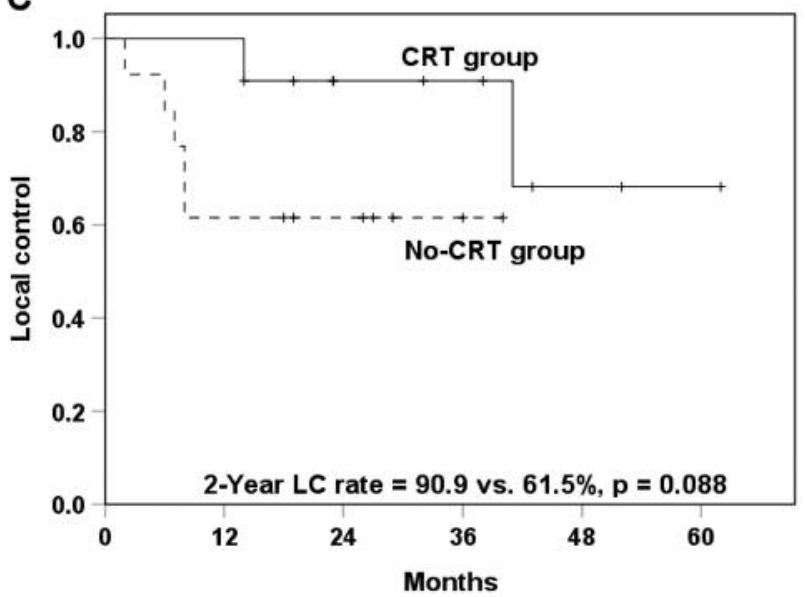

Figure 1. Kaplan-Meier curves of overall survival (OS) (A), diseasefree survival $(D F S)(B)$, and local control $(C)$ in association with concurrent chemoradiotherapy $(C R T)(n=11)$ and no-CRT $(n=13)$.
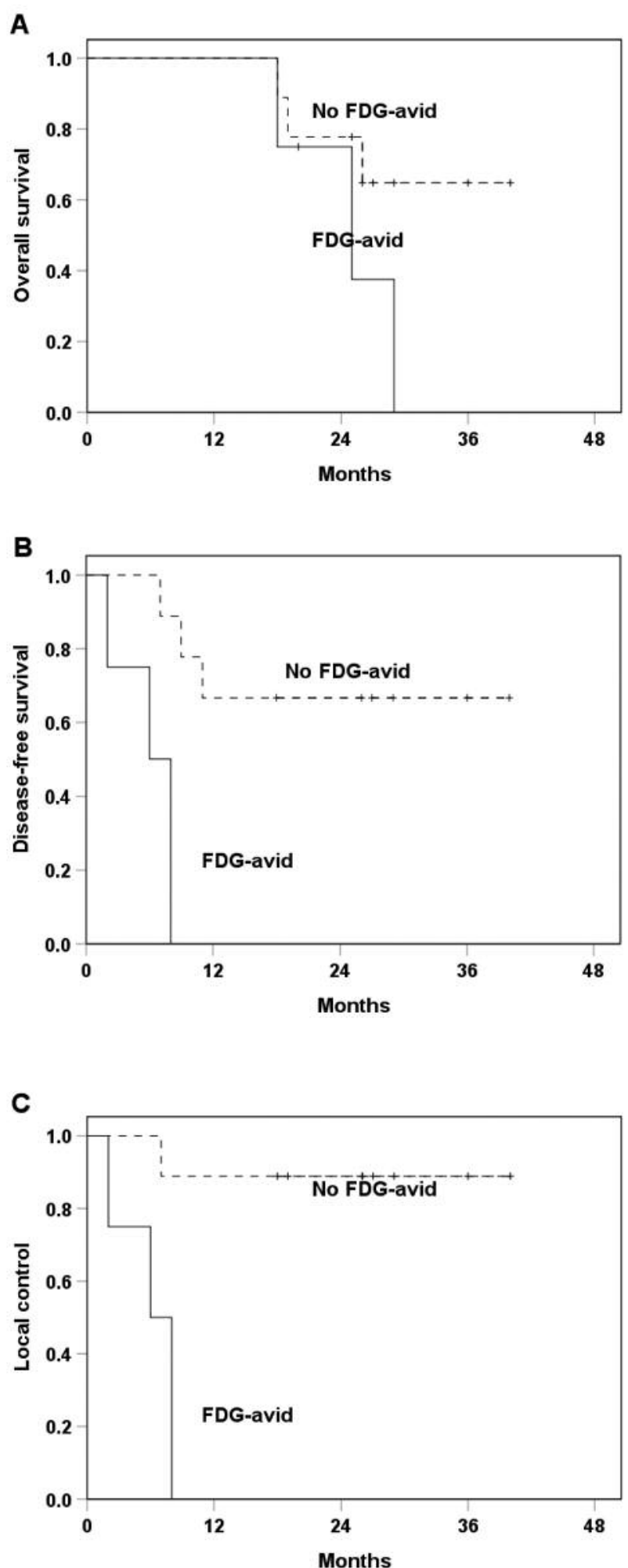

Figure 2. Kaplan-Meier curves of overall survival (OS) (A), diseasefree survival (DFS) (B), and local control $(C)$ in association with the presence of ${ }^{18}$ F-fluorodeoxyglucose $(F D G)$-avid $(n=4)$ and non FDGavid $(n=9)$ primary tumors in the no concurrent chemoradiotherapy group. 
Table II. Univariate analysis of 2-year overall survival (OS), disease-free survival (DFS), and local control (LC) in the group treated without concurrent chemotherapy $(n=13)$.

\begin{tabular}{|c|c|c|c|c|c|c|c|c|}
\hline Factor & & Patients (n) & OS $(\%)$ & $p$-Value & DFS $(\%)$ & $p$-Value & LC $(\%)$ & $p$-Value \\
\hline \multirow[t]{2}{*}{ Age* } & $<77$ Years & 4 & 50 & 0.742 & 75 & 0.137 & 100 & 0.084 \\
\hline & $\geq 77$ Years & 9 & 88.9 & & 33.3 & & 44.4 & \\
\hline \multirow[t]{2}{*}{ Gender } & Female & 1 & 100 & 0.449 & 100 & 0.362 & 100 & 0.47 \\
\hline & Male & 12 & 75 & & 41.7 & & 58.3 & \\
\hline \multirow[t]{2}{*}{ Lymph node involvement } & No & 11 & 72.7 & 0.998 & 45.5 & 0.769 & 54.5 & 0.282 \\
\hline & Yes & 2 & 100 & & 50 & & 100 & \\
\hline \multirow[t]{2}{*}{ Tumor length* } & $<5.5 \mathrm{~cm}$ & 6 & 83.3 & 0.895 & 50 & 0.482 & 83.3 & 0.115 \\
\hline & $\geq 5.5 \mathrm{~cm}$ & 7 & 71.4 & & 42.9 & & 42.9 & \\
\hline \multirow[t]{2}{*}{ FDG-avid primary tumor } & No & 9 & 77.8 & 0.148 & 66.7 & 0.002 & 88.9 & 0.002 \\
\hline & Yes & 4 & 75 & & 0 & & 0 & \\
\hline \multirow[t]{2}{*}{ Symptomatic } & No & 10 & 70 & 0.893 & 50 & 0.447 & 70 & 0.238 \\
\hline & Yes & 3 & 100 & & 33.3 & & 33.3 & \\
\hline \multirow[t]{2}{*}{ External dose* } & $<59.4$ Gy & 3 & 100 & 0.224 & 33.3 & 0.361 & 33.3 & 0.166 \\
\hline & $\geq 59.4 \mathrm{~Gy}$ & 10 & 70 & & 50 & & 70 & \\
\hline \multirow{2}{*}{ Sequential chemotherapy } & No & 6 & 50 & 0.026 & 33.3 & 0.503 & 50 & 0.54 \\
\hline & Yes & 7 & 100 & & 57.1 & & 71.4 & \\
\hline
\end{tabular}

FDG: ${ }^{18}$ F-Fluorodeoxyglucose. ${ }^{*}$ Median values were used as the optimal cut-off to discriminate the low- and high-probability categories.

We did not treat those aged 75 years or more with concurrent chemotherapy, as little is known about the outcome of CRT for elderly patients with T1 EC. Takeuchi et al. compared the outcome of CRT based on 5 -fluorouracil/cisplatin and $60 \mathrm{~Gy}$ of radiation between 33 elderly (>70 years) and 145 non-elderly patients with stage II-III (non-T4) SCC EC. A significantly poorer survival rate, and higher CRT discontinuation and grade 3 or more leucopenia rates were reported in elderly patients (7). They suggested that lower treatment compliance in elderly patients might be the major reason for the inferior outcomes.

We used ME-NBI for diagnosing the tumor depth according to Inoue's classification (9). Recently, the accuracy of endoscopic ultrasound has been questioned for T1 stage EC $(14,15)$ and the superiority of ME-NBI has been shown (16). All our endoscopic procedures were performed by endoscopists with extensive experience in this field and staged as T1. Primary tumors were not visible by esophagography or contrast-enhanced CT.

There are a few articles demonstrating the utility of FDGPET/CT in staging for patients with T1 EC treated with esophagectomy. FDG avidity and SUVmax were reported as significant preoperative predictors as they were used to detect T1 EC tumors infiltrating the middle or deep submucosal layer $(17,18)$. Most of these patients had SCC. Furukawa et al. analyzed 40 patients with T1 EC treated with esophagectomy, and the optimal SUVmax cutoff required to predict pathological tumor invasion to the middle submucosal layer or beyond was 2.7. Furthermore, SUVmax $\geq 2.7$ of the primary tumor on FDG-PET was associated with poor DFS and disease-specific survival (17), whereas for adenocarcinoma, FDG-PET/CT was not indicated as being useful for staging T1 EC. Little et al. found that although FDG uptake and SUV increased with the increasing depth of tumor invasion, FDG-PET could not differentiate pTis from pT1. Their patients with tumor uptake had SUVmax ranging from 2.1 to 16.6 , with a mean of 3.9 and median of 3.5 (19). In present study, SUVmax ranged from 2.8 to 5.1 , with a mean of 3.7 and median of 3.6 .

This was a single-center retrospective study and had several limitations, including a relatively small number of patients. We were unable to draw conclusions as to why the OS rate at 2 years was significantly higher for patients with $\mathrm{SCT}$ in the no-CRT group. There were also other factors, such as the LN status, reported to be unfavorable prognostic factors in patients with EC treated with CRT (20), but the number of our patients was limited and it was not possible to evaluate these factors by multivariate analysis. A poor nutritional status, based on the serum albumin level and body weight at the beginning of radiotherapy, was also reported to be a prognostic factor affecting both DFS and OS (21). Our patients had a good nutritional status as their albumin levels were above $3.6 \mathrm{mg} / \mathrm{dl}$ and they showed no weight loss.

In conclusion, DFS and LC at 2 years were significantly lower in patients with FDG-avid primary tumor in the no-CRT group. Although this was a small group study, it is important to note that all patients with FDG-avid primary tumor in the no-CRT group developed local recurrence. To our knowledge, this is the first report to suggest that FDG-PET/CT plays a clinically significant role in the treatment of patients with $\mathrm{T} 1$ 
EC undergoing radiotherapy. Large, prospective, multiinstitutional studies are needed to confirm our results.

\section{Compliance with Ethical Standards}

This study was approved by the Institutional Committee (approval number: B170700047). For this retrospective type of study, formal consent of patients was not required.

\section{Conflicts of Interest}

The Authors declare that there is no conflict of interest in regard to this study.

\section{References}

1 Korn WM: Prevention and management of early esophageal cancer. Curr Treat Options Oncol 5: 405-416, 2004.

2 Leers JM, DeMeester SR, Oezcelik A, Klipfel N, Ayazi S, Abate E, Zehetner J, Lipham JC, Chan L, Hagen JA and DeMeester TR: The prevalence of lymph node metastases in patients with T1 esophageal adenocarcinoma a retrospective review of esophagectomy specimens. Ann Surg 253: 271-278, 2011.

3 Rice TW, Ishwaran H, Hofstetter WL, Kelsen DP, AppersonHansen C, Blackstone EH and Worldwide Esophageal Cancer Collaboration I: Recommendations for pathologic staging (pTNM) of cancer of the esophagus and esophagogastric junction for the 8th edition AJCC/UICC staging manuals. Dis Esophagus 29: 897-905, 2016.

4 Karaosmanoglu AD and Blake MA: Applications of PET-CT in patients with esophageal cancer. Diagn Interv Radiol 18: 171182, 2012.

5 Al-Sarraf M, Martz K, Herskovic A, Leichman L, Brindle JS, Vaitkevicius VK, Cooper J, Byhardt R, Davis L and Emami B: Progress report of combined chemoradiotherapy versus radiotherapy alone in patients with esophageal cancer: An Intergroup study. J Clin Oncol 15: 277-284, 1997.

6 Cooper JS, Guo MD, Herskovic A, Macdonald JS, Martenson JA Jr., Al-Sarraf M, Byhardt R, Russell AH, Beitler JJ, Spencer S, Asbell SO, Graham MV and Leichman LL: Chemoradiotherapy of locally advanced esophageal cancer: long-term follow-up of a prospective randomized trial (RTOG 85-01). Radiation Therapy Oncology Group. JAMA 281: 1623-1627, 1999.

7 Takeuchi S, Ohtsu A, Doi T, Kojima T, Minashi K, Mera K, Yano T, Tahara M, Muto M and Nihei K: A retrospective study of definitive chemoradiotherapy for elderly patients with esophageal cancer. Am J Clin Oncol 30: 607-611, 2007.

8 Ogino I, Watanabe S, Sakamaki K, Ogino Y, Kunisaki C and Kimura K: Dosimetric predictors of radiation-induced pericardial effusion in esophageal cancer. Strahlenther Onkol 193: 552-560, 2017.

9 Inoue H, Kaga M, Ikeda H, Sato C, Sato H, Minami H, Santi EG, Hayee B and Eleftheriadis N: Magnification endoscopy in esophageal squamous cell carcinoma: a review of the intrapapillary capillary loop classification. Ann Gastroenterol 28: 41-48, 2015.

10 Koide Y, Kodaira T, Tachibana H, Tomita N, Makita C, Itoh M, Abe T, Muro K, Tajika M, Niwa Y, Itoh Y and Naganawa S: Clinical outcome of definitive radiation therapy for superficial esophageal cancer. Jpn J Clin Oncol 47: 393-400, 2017.
11 Lee DY, Moon SH, Cho KH, Kim TH, Kim MS, Lee JY and Suh YG: Treatment outcomes of extended-field radiation therapy for thoracic superficial esophageal cancer. Radiat Oncol J 35: 241248, 2017.

12 Murakami Y, Nagata Y, Nishibuchi I, Kimura T, Kenjo M, Kaneyasu Y, Okabe T, Hashimoto Y and Akagi Y: Long-term outcomes of intraluminal brachytherapy in combination with external beam radiotherapy for superficial esophageal cancer. Int J Clin Oncol 17: 263-271, 2012.

13 Nemoto K, Matsumoto Y, Yamakawa M, Jo S, Ito Y, Oguchi M, Kokubo N, Nishimura Y, Yamada S and Okawa T: Treatment of superficial esophageal cancer by external radiation therapy alone: results of a multi-institutional experience. Int J Radiat Oncol Biol Phys 46: 921-925, 2000.

14 Bergeron EJ, Lin J, Chang AC, Orringer MB and Reddy RM: Endoscopic ultrasound is inadequate to determine which T1/T2 esophageal tumors are candidates for endoluminal therapies. J Thorac Cardiovasc Surg 147: 765-771, 2014.

15 Tekola BD, Sauer BG, Wang AY, White GE and Shami VM: Accuracy of endoscopic ultrasound in the diagnosis of T2N0 esophageal cancer. J Gastrointest Cancer 45: 342-346, 2014.

16 Mizumoto T, Hiyama T, Oka S, Yorita N, Kuroki K, Kurihara M, Yoshifuku Y, Sanomura Y, Urabe Y, Arihiro K, Tanaka S and Chayama K: Diagnosis of superficial esophageal squamous cell carcinoma invasion depth before endoscopic submucosal dissection. Dis Esophagus, 2017. doi: 10.1093/dote/dox 142 . [Epub ahead of print]

17 Furukawa T, Hamai Y, Hihara J, Emi M, Yamakita I, Ibuki Y and Okada M: Clinical significance of FDG-PET to predict pathologic tumor invasion and lymph node metastasis of superficial esophageal squamous cell carcinoma. Ann Surg Oncol 23: 4086-4092, 2016.

18 Miyata H, Doki Y, Yasuda T, Yamasaki M, Higuchi I, Makari Y, Matsuyama J, Hirao T, Takiguchi S, Fujiwara Y and Monden M: Evaluation of clinical significance of ${ }^{18} \mathrm{~F}$-fluorodeoxyglucose positron emission tomography in superficial squamous cell carcinomas of the thoracic esophagus. Dis Esophagus 21: 144$150,2008$.

19 Little SG, Rice TW, Bybel B, Mason DP, Murthy SC, Falk GW, Rybicki LA and Blackstone EH: Is FDG-PET indicated for superficial esophageal cancer? Eur J Cardiothorac Surg 31: 791796, 2007.

20 Jeene PM, Versteijne E, van Berge Henegouwen MI, Bergmann JJ, Geijsen ED, van Laarhoven $\mathrm{HW}$ and Hulshof MC: Supraclavicular node disease is not an independent prognostic factor for survival of esophageal cancer patients treated with definitive chemoradiation. Acta Oncol 56: 33-38, 2017.

21 Clavier JB, Antoni D, Atlani D, Ben Abdelghani M, Schumacher C, Dufour P, Kurtz JE and Noel G: Baseline nutritional status is prognostic factor after definitive radiochemotherapy for esophageal cancer. Dis Esophagus 27: 560-567, 2014. 